\title{
Providing Quality Primary Care to Older Adults
}

\author{
Gregg Warshaw, MD
}

Family physicians are a critical source of primary care for older Americans; elderly people living in rural areas are particularly dependent on family physicians for primary care. ${ }^{1}$ However, there is a trend for older adults to seek care from non-primary care specialists at a higher rate than in the recent past. In $2005,43 \%$ of ambulatory care visits by patients aged 65 and over were to primary care physicians and $57 \%$ were to non-primary care specialists. In 1980, $62 \%$ of ambulatory care visits by patients aged 65 and over were to primary care physicians and $38 \%$ were to non-primary care specialists. ${ }^{2}$ The expanded use of subspecialists by the Medicare population is particularly striking among older adults with multiple chronic illnesses. During 1999, older adults with 5 or more chronic illnesses received medical services from a mean of 13.8 unique physicians. ${ }^{3}$ This trend away from the traditional role of the primary care physician in the care of older adults places these patients at risk for poor continuity of care, adverse drug events, and the iatrogenic consequences of the over-utilization of diagnostic and therapeutic interventions. To improve the quality and control the costs of medical care for older adults, primary care physicians, and especially family physicians, must reassert their central role in the care of older adults.

This issue of the Fournal of the American Board of Family Medicine includes several articles that provide specific new findings that can help inform the care of older patients in the family physician's office. These articles also illustrate some key principles that can lead to improved primary care for older patients.

Submitted 9 March 2009; revised 9 March 2009; accepted 9 March 2009.

From the Department of Family Medicine; University of Cincinnati College of Medicine, Ohio.

Funding: none.

Conflict of interest: none declared.

Corresponding author: Gregg Warshaw, MD, Department of Family Medicine, University of Cincinnati College of Medicine, P.O. Box 670504, Cincinnati, OH 45267-0504 (E-mail: Gregg.Warshaw@uc.edu).
Two articles address the vexing problems of when and how to screen for prostate cancer in older men and what is the best treatment approach for localized prostate cancer. Prostate cancer is the second leading cause of cancer deaths among men in the United States. The incidence increases with age and is rare in men younger than 40. In autopsy studies in which the entire prostate is examined, incidental histologic evidence of prostate cancer is found in $80 \%$ of men older than $80 .{ }^{4}$ Using practice-based research networks in the Northeast, Hudson and colleagues ${ }^{5}$ studied the use of the prostate-specific antigen (PSA) screening test among middle-aged and older men. This study found that men aged 75 years and older are receiving PSA testing at a rate of $74.6 \%$, which is comparable to the screening rates for men aged 50 to 74 years. The authors point out that this high rate of screening among older men is not consistent with evidence-based practice guidelines. Of most interest, the authors conclude that PSA screening rates were lower in offices with more effective intraoffice communication among office staff and between staff and patients.

No direct evidence exists to show that early detection decreases prostate cancer mortality rates. ${ }^{4}$ Not all prostate cancer in older men needs to be diagnosed because the majority of older men with prostate cancer die with the disease, not from it. The ability of an intervention directed toward someone already old to prevent future disability is handicapped by the "narrow therapeutic window" that characterizes the older person. Prevention in the elderly must also account for their susceptibility to the iatrogenic consequences of the best intended actions. Practice guidelines are in agreement that the controversy surrounding prostate cancer screening should be discussed with patients to achieve individualized, informed courses of action. The family physicians office is an ideal setting for this conversation.

In a related article, Mohan and colleagues ${ }^{6}$ ex- $^{-}$ plore prostate cancer patients' knowledge about 
survival expectations with active treatment versus watchful waiting. The researchers surveyed 184 men, newly diagnosed with screen-detected localized prostate cancer, from a large urology practice in Norfolk, Virginia. Forty-nine percent of the men in the study were 60 to 70 years old and $11.9 \%$ were older than 70 years. Twenty-two percent of these older patients chose watchful waiting. The researchers found that these 184 men underestimated their life expectancy without treatment and overestimated the survival benefit of treatment. The authors conclude that these patients are not receiving adequate information to make informed treatment decisions. Localized prostate cancer provides a potential for curative interventions. However, the prevalence of men dying with prostate cancer (often asymptomatic), but not from the disease, suggests that older men are not benefiting from treatment for localized prostate cancer. No clinical trials have shown that treatment prolongs life in older men with localized prostate cancer when treatment is compared with watchful waiting. Watchful waiting is a reasonable treatment approach to offer men with less than a 10 -year life expectancy, those who have significant medical comorbidities, or whose tumor is small and well to moderately differentiated.

The watchful waiting approach recognizes the potential for functional decline in older men treated aggressively for localized prostate cancer. Men are more likely to experience impotence and stress incontinence after radical prostatectomy, with symptoms ranging from occasional leakage to no urinary control. The incidence of urinary stress incontinence after radiation therapy is significantly less than with surgery, but the presence of irritative voiding dysfunction is greater. Brachytherapy involves retropubic or perineal implantation of radioactive seeds. Urinary symptoms after brachytherapy include increased frequency, dysuria, and urge incontinence. ${ }^{7}$ Once again, the family physician's office is an ideal setting for educational interventions directed at older men diagnosed with localized prostate cancer.

Another valuable article in this issue of the $7 A B F M$ provides new information about the characteristics of uncomplicated urinary tract infections (UTI) in ambulatory older women. Grover and colleagues ${ }^{8}$ examined the characteristics of UTIs in the family medicine center associated with the Department of Family Medicine, Mayo Clinic, Scottsdale, Arizona. One hundred six older women with UTIs were identified out of 228 UTI cases. Fifty-nine of these older women had medically uncomplicated UTIs whereas 47 were complicated. The presence of Escherichia coli as the cultured pathogen was more likely in uncomplicated patients of all ages, and older age was not independently associated with the presence of other pathogens. It seemed that trimethoprim-sulfamethoxazole (TMP-SMX) would have successfully treated $86 \%$ of the E. coli infections, although in this study ciprofloxacin was more likely to be prescribed for older women. The use of ciprofloxacin was based on practice recommendations from a previous study that included a diverse population of older women, including those living in nursing homes. ${ }^{9}$ The authors conclude that, in their office setting, empirical treatment with TMP-SMX may be effective treatment for uncomplicated UTIs in older women.

Extrapolating treatment guidelines developed for younger patients to older adults requires caution. Previous clinical trials have frequently excluded older adults. The risk exists that older adults will receive inadequate or ineffective therapy. ${ }^{10} \mathrm{On}$ the other hand, healthy older adults with common ambulatory problems may not require a more aggressive treatment approach.

Among older women, lower UTI (cystitis), characterized by dysuria, increased frequency, and urgency (not fever, which generally indicates upper tract disease), may be treated with 3 days of therapy. ${ }^{11}$ Previous clinical trials in older women indicate that fluoroquinolones are more efficacious than TMP-SMX, likely because of E. coli TMPSMX resistance rates of $10 \%$ to $20 \%$ in most areas of the country. The Grover study suggests that, among a community outpatient practice, TMPSMX may be adequate first-line therapy. Other reasonable choices in some settings include amoxicillin (particularly for enterococcal infection) and first-generation cephalosporins in patients with multiple antibiotic intolerances. Culture is not required unless first-line therapy fails.

Complicated UTI, characterized by fever, chills, nausea, and flank pain, and/or accompanied by lower tract symptoms, requires therapy for 7 to 21 days. Because of the excellent bioavailability of many antibiotics, particularly the fluoroquinolones, intravenous therapy is not essential if the patient can tolerate oral medications. Culture and sensitivity data are useful in guiding therapy for complicated UTIs and should be obtained in most cases. ${ }^{12}$ 
The articles discussed above as well as several other contributions in this issue of the $7 A B F M$ add to the medical knowledge base that is essential to providing quality care to older adults. The aging of America over the coming decades will lead to increasing numbers of older people seeking care from family physicians. The development of the medical home and application of the chronic disease model will significantly benefit the older adult. ${ }^{13,14}$ The family physician cannot do it all. Successful care for older adults will require teamwork with other medical specialists, nurses, social workers, health educators, and the therapy professions, to name a few. However, one health professional must be responsible for the overall plan and coordination of care, and the family physician is often best suited for this task. It can be burdensome and challenging, but without such leadership, the older patient cannot be assured of optimal care.

\section{References}

1. Mold JW, Fryer GE, Phillips RL Jr, Dovey SM, Green LA. Family physicians are the main source of primary health care for the Medicare population. Am Fam Physician 2002;66:2032.

2. US Department of Health and Human Services, National Center for Health Statistics. National Ambulatory Medical Care Survey, 2004. Hyattsville (MD): US Department of Health and Human Services, National Center for Health Statistics; 2006.

3. Anderson GF. Medicare and chronic conditions. N Engl J Med 2005;353:305-9.

4. Harris R, Lohr KN. Screening for prostate cancer: an update of the evidence for the US Preventive Services Task Force. Ann Intern Med 2002;137:91729.
5. Hudson SV, Ohman-Strickland P, Ferrante JM, LuYao G, Orzano AJ, Crabtree BF. Prostate-specific antigen testing among the elderly in communitybased family medicine practices. J Am Board Fam Med 2009;22:257-65.

6. Mohan R, Beydoun H, Barnes-Ely ML, et al. Patients' survival expectations before localized prostate cancer treatment by treatment status. J Am Board Fam Med 2009;22:247-56.

7. Penson DF, Litwin MS. The physical burden of prostate cancer. Urol Clin North Am 2003;30:30513.

8. Grover ML, Bracamonte JD, Kanodia AK, Edwards $\mathrm{FD}$, Weaver AL. Urinary tract infection in women over the age of 65: is age alone a marker of complication? J Am Board Fam Med 2009;22:266-71.

9. Gomolin IH, Siami PF, Reuning-Scherer J, Haverstock DC, Heyd A; Oral Suspension Study Group. Efficacy and safety of ciprofloxacin oral suspension versus trimethoprim-sulfamethoxazole oral suspension for treatment of older women with acute urinary tract infection. J Am Geriatr Soc 2001;49:1606-13.

10. Tinetti ME, Bogardus ST, Agostini JV. Potential pitfalls of disease-specific guidelines for patients with multiple conditions. N Engl J Med 2004;351:2870-3.

11. Lutters M, Vogt N. Antibiotic duration for treating uncomplicated, symptomatic lower urinary tract infections in elderly women. Cochrane Database Syst Rev 2002;(3):CD001535.

12. High KP. Infectious diseases. Geriatrics Review Syllabus, 6th edition. New York: American Geriatrics Society; 2006.

13. Rogers JC. The patient-centered medical home movement-promise and peril for family medicine. J Am Board Fam Med 2008;21:370-4.

14. Rosenthal TC. The medical home: growing evidence to support a new approach to primary care. J Am Board Fam Med 2008;21:427-40. 